\title{
The gauge theorem for a class of additive functionals of zero energy
}

\author{
Joseph Glover ${ }^{\star}$, Murali Rao, and Renming Song \\ Department of Mathematics, University of Florida, Gainesville, FL 32611, USA
}

Received February 27, 1992; in revised form April 6, 1993

Summary. In earlier works, the gauge theorem was proved for additive functionals of Brownian motion of the form $\int_{0}^{t} q\left(B_{s}\right) d s$, where $q$ is a function in the Kato class. Subsequently, the theorem was extended to additive functionals with Revuz measures $\mu$ in the Kato class. We prove that the gauge theorem holds for a large class of additive functionals of zero energy which are, in general, of unbounded variation. These additive functionals may not be semi-martingales, but correspond to a collection of distributions that belong to the Kato class in a suitable sense. Our gauge theorem generalizes the earlier versions of the gauge theorem.

Mathematics Subject Classification (1991): 60J65, 60J55, 60J57

Brownian motion has long been used to treat the Dirichlet problem and other differential equations involving the Laplacian in a probabilistic manner. In resolving the Dirichlet problem, one tries to solve $\Delta u=0$ on some bounded open domain $D$ subject to the condition $u=f$ on the boundary of $D$, for some specified function $f$. Kac showed that the probabilistic approach can be extended to treat differential operators of the form $\Delta-q$, or more generally, of the form $\Delta-\mu$ by considering Brownian motion "killed" by a multiplicative functional associated with $q$ or $\mu$. For example, the formal solution of $\Delta u-q u=0, u=f$ on the boundary of $D$ is given by

$$
E^{x}\left[f\left(X\left(\tau_{D}\right)\right) \exp \left(A\left(\tau_{D}\right)\right)\right]
$$

where $\tau_{D}$ is the first exit time of the Brownian motion $X_{t}$ from $D$ and where

$$
A_{t}=\int_{0}^{t} q\left(X_{s}\right) d s .
$$

* Research supported in part by NSA grant MDA-92-H-30324 
In order to make this precise, however, one must check that $\exp \left(A\left(\tau_{D}\right)\right)$ is integrable. Chung and Rao initiated the study of this problem for one dimensional Brownian motion, and since then, many authors have examined this question for Brownian motion in $R^{d}$ and for more general Markov processes. See for example, $[1,3,5,6,7,9,13,14]$ and the references therein. The basic result has come to be known as the gauge theorem, and it can be stated for Brownian motion as follows. Gauge theorem. Let $\mu$ be a measure on $R^{d}$ belonging to the Kato class and let $A_{t}$ be the continuous additive functional of $X_{t}$ associated with $\mu$. If $D$ is a bounded, connected open subset of $R^{d}$, then the function

$$
g(x)=E^{x}\left\{\exp \left(A\left(\tau_{D}\right)\right)\right\}
$$

is either bounded or identically infinite on $D$, where $\tau_{D}=\inf \left\{t>0: X_{t} \notin D\right\}$.

The assumption that $\mu$ is in the Kato class implies that its associated additive functional $A_{t}$ is of bounded variation: this is crucial in all the arguments in the references above since $A_{t}$ is then the distribution function of a signed measure. However, there are many naturally occurring additive functionals which are not of bounded variation. How can these be investigated, and is there still a gauge theorem?

In this article, we prove the gauge theorem holds for a large class of continuous additive functionals of zero energy which are generally not of bounded variation (although our gauge theorem includes the one stated above as a special case). See Theorem 3.8. Interestingly, our additive functionals are not even semimartingales, so stochastic calculus is of little use in this context.

The gauge theorem is formulated and proved only for a bounded domain $D$ in this paper, but the argument, with some minor changes, can be used to prove a gauge theorem for any domain $D$ of finite Lebesgue measure.

The contents of this paper are organized as follows: Sect. 1 serves as a preparation. It contains the necessary definitions and some basic results (the only new result in this section is perhaps Theorem 1.9). Section 2 contains some important properties of the class of additive functionals under study, and these properties will be crucial in proving the gauge theorem. Section 3 contains the gauge theorem and some important consequences of it.

In a subsequent article, we shall study the generalized Schrödinger semigroups corresponding to this general class of additive functionals and apply the gauge theorem to solve the Dirichlet problem associated with generalized Schrödinger operators.

\section{Preliminaries}

In this paper we shall always assume that $X=\left(\Omega, \mathscr{F}, \mathscr{F}_{t}, X_{t}, \theta_{t}, P^{x}\right)$ is a standard Brownian motion in $R^{d}, d \geqq 3$, and that $\left(\mathscr{E}, H^{1}\left(R^{d}\right)\right)$ is the regular Dirichlet form associated with $X$. The Sobolev space $H^{1}\left(R^{d}\right)$ is defined by

$$
H^{1}\left(R^{d}\right)=\left\{u \in L^{2}\left(R^{d}\right): \frac{\partial u}{\partial x_{i}} \in L^{2}\left(R^{d}\right), i=1,2, \ldots, d\right\}
$$

and $\mathscr{E}$ is defined by

$$
\mathscr{E}(u, v)=\frac{1}{2} \int_{R^{d}} \nabla u \cdot \nabla v d x, \quad \forall u, v \in H^{1}\left(R^{d}\right) .
$$


For a positive number $\alpha$, we shall write

$$
\mathscr{E}_{\alpha}(u, v)=\mathscr{E}(u, v)+\alpha(u, v),
$$

where $(u, v)$ denotes the inner product in $L^{2}\left(R^{d}\right)$.

As usual we use $p(t, x, y)$ to denote transition density of $X_{t}$. We shall sometimes write

$$
P_{t} \mu(x)=\int_{R^{d}} p(t, x, y) \mu(d y)
$$

and

$$
G_{\alpha} \mu(x)=\int_{0}^{\infty} e^{-\alpha t} P_{t} \mu(x) d t
$$

provided the right hand sides make sense.

(1.1) Definition. A signed Radon measure $\mu$ on $R^{d}$ is said to be in the Kato class if

$$
\lim _{r \downarrow 0} \sup _{x \in R^{d}} \int_{|x-y|<r} \frac{\mu^{*}(d y)}{|x-y|^{d-2}}=0
$$

where $\mu^{*}=\mu^{+}+\mu^{-}$with $\mu^{+}$and $\mu^{-}$being the positive part and the negative part of $\mu$ respectively. In the sequel we shall use $K_{d}$ to denote the Kato class.

It is easy to see from the definition above that a signed Radon measure is in $K_{d}$ if and only if both $\mu^{+}$and $\mu^{-}$are in $K_{d}$.

The following result is well known. See for example, [2].

(1.2) Proposition. Let $\mu$ be a Radon measure on $R^{d}$. Then $\mu \in K_{d}$ if and only if

$$
\lim \sup _{t \downarrow 0} \int_{x \in R^{d}}^{t} \int_{R^{d}} p(s, x, y) \mu^{*}(d y) d s=0 .
$$

(1.3) Definition. A (nonnegative) Radon measure $\mu$ on $R^{d}$ is said to be of finite energy integral if there is a constant $C>0$ such that

$$
\int_{R^{a}}|v(x)| \mu(d x) \leqq C \sqrt{\mathscr{E}_{1}(v, v)}, \quad \forall v \in C_{0}^{\infty}\left(R^{d}\right)
$$

where $C_{0}^{\infty}\left(R^{d}\right)$ is the collection of all infinitely differentiable functions with compact support on $R^{d}$. We shall use $S_{0}$ to denote the collection of measures of finite energy integrals.

By the Riesz representation theorem, a Radon measure $\mu \in S_{0}$ if and only if there exists an element $U_{1} \mu \in H^{1}\left(R^{d}\right)$ such that

$$
\mathscr{E}_{1}\left(U_{1} \mu, v\right)=\int_{R^{d}} v(x) \mu(d x), \quad \forall v \in C_{0}^{\infty}\left(R^{d}\right) .
$$

(1.4) Lemma. Let $\mu$ be a Radon measure which is in $K_{d}$. Then for any compact subset $F$ of $R^{d}$, the restriction of $\mu$ to $F, 1_{F} \cdot \mu$, is of finite energy integral.

Proof. From (1.2) it is easy to show that

$$
\sup _{x \in R^{d}} \int_{0}^{\infty} e^{-s} P_{s} \mu(x) d s<\infty .
$$


In particular, the following function

$$
G_{1}\left(1_{F} \cdot \mu\right)(x)=\int_{0}^{\infty} e^{-s} P_{s}\left(1_{F} \cdot \mu\right)(x) d s
$$

is bounded, say, by $M$. Now

$$
\int_{R^{d}}\left(G_{1}\left(1_{F} \cdot \mu\right)(x)\right)^{2} d x \leqq M \int_{R^{d}} \int_{0}^{\infty} e^{-s} P_{s}\left(1_{F} \cdot \mu\right)(x) d s d x \leqq M \mu(F),
$$

so $G_{1}\left(1_{F} \cdot \mu\right) \in L^{2}\left(R^{d}\right)$. And since

$$
\begin{aligned}
\lim _{t \downarrow 0} \frac{1}{t} & \left(G_{1}\left(1_{F} \cdot \mu\right)-e^{-t} P_{t}\left(G_{1}\left(1_{F} \cdot \mu\right)\right), G_{1}\left(1_{F} \cdot \mu\right)\right) \\
& \leqq \lim _{t \downarrow 0} \frac{M}{t} \int_{R^{d}} \int_{0}^{t} e^{-s} P_{s}\left(1_{F} \cdot \mu\right)(x) d s d x \\
& \leqq M \mu(F)<\infty,
\end{aligned}
$$

by Lemma 1.3 .4 of $[10]$ we know that $G_{1}\left(1_{F} \cdot \mu\right) \in H^{1}\left(R^{d}\right)$. Therefore, for any $v \in C_{0}^{\infty}\left(R^{d}\right)$,

$$
\begin{aligned}
\mathscr{E}_{1}\left(G_{1}\left(1_{F} \cdot \mu\right), v\right) & =\lim _{t \downarrow 0} \frac{1}{t}\left(G_{1}\left(1_{F} \cdot \mu\right)-e^{-t} P_{t}\left(G_{1}\left(1_{F} \cdot \mu\right)\right), v\right) \\
& =\lim _{t \downarrow 0}\left(\frac{1}{t} \int_{0}^{t} e^{-s} P_{s}\left(1_{F} \cdot \mu\right) d s, v\right) \\
& =\int_{R^{d}} v(x)\left(1_{F} \cdot \mu\right)(d x)
\end{aligned}
$$

Thus $1_{F} \cdot \mu \in S_{0}$.

(1.5) Corollary. If a Radon measure $\mu$ is in $K_{d}$, then $\mu$ does not charge polar sets. Therefore it follows from [12] that for any Radon measure $\mu$ in $K_{d}$, there exists a unique positive continuous additive functional $A=\left(A_{t}\right)_{t \geqq 0}$ of $X$ such that

$$
\lim _{t \downarrow 0} \frac{1}{t} \int_{R^{d}} h(x) E^{x} \int_{0}^{t} f\left(X_{s}\right) d A_{s} d x=\int_{R^{d}} h(x) f(x) \mu(d x)
$$

for all $\gamma$-excessive $\left(\gamma \geqq 0\right.$ ) functions $h$ and all nonnegative Borel functions $f$ on $R^{d}$.

Suppose now that $\mu \in K_{d}$. Let $\left(A_{t}^{+}\right)$and $\left(A_{t}^{-}\right)$be the positive additive functionals associated with $\mu^{+}$and $\mu^{-}$respectively in the manner of (1.6). If we set $A_{t}=A_{t}^{+}-A_{t}^{-}$, then $\left(A_{t}\right)$ is a continuous additive functional of bounded variation. We shall call $\left(A_{t}\right)$ the continuous additive functional associated with $\mu$.

Let $u \in H^{1}\left(R^{d}\right)$ be bounded and continuous. Then from [10] we know that

$$
A_{t}^{[u]}=u\left(X_{t}\right)-u\left(X_{0}\right)-\int_{0}^{t} \nabla u\left(X_{s}\right) \cdot d X_{s}
$$

is a continuous additive functional of zero energy. In general, $A_{t}^{[u]}$ is not of bounded variation. We shall call $\left(A_{t}^{[u]}\right)$ the continuous additive functional of zero energy generated by $u$. 
(1.8) Definition. A bounded continuous function $u \in H^{1}\left(R^{d}\right)$ is said to be admissible if $|\nabla u(x)|^{2} d x \in K_{d}$.

Remark. If one wishes to prove the gauge theorem for a domain which is only assumed to be of finite Lebesgue measure, the continuity of $u$ in the definition above should be strengthened to uniform continuity.

(1.9) Theorem. Let $\mu \in K_{d}$ be of compact support and let $\left(A_{t}\right)$ be the continuous additive functional associated with $\mu$. Then there is an admissible $u$ such that $A_{t}=A_{t}^{[u]}$.

Proof. Without loss of generality we can assume that $\mu$ is nonnegative. Since for any $t>0$

$$
\begin{aligned}
\int_{R^{d}} E^{x} A_{t}^{2} d x & =2 \int_{R^{d}} E^{x} \int_{0}^{t} E^{X_{s}} A_{t-s} d A_{s} d x \\
& \leqq 2\left(\sup _{x \in R^{d}} E^{x} A_{t}\right) \cdot \int_{R^{d}} E^{x} A_{t} d x \\
& =2 t \mu\left(R^{d}\right) \cdot\left(\sup _{x \in R^{d}} E^{x} A_{t}\right),
\end{aligned}
$$

we know that

$$
\lim _{t \downarrow 0} \frac{1}{2 t} \int_{R^{d}} E^{x} A_{t}^{2} d x=0,
$$

or equivalently, $A_{t}$ is of zero energy. Put

$$
u(x)=-E^{x} A_{\infty}=-G \mu(x) .
$$

Then $u \in H^{1}\left(R^{d}\right)$ and it is bounded and continuous. Furthermore it tends to zero at infinity, so it is uniformly continuous. It is easy to see that

$$
u\left(X_{t}\right)-u\left(X_{0}\right)=\left(-E^{x}\left[A_{\infty} \mid \mathscr{F}_{t}\right]-u\left(X_{0}\right)\right)+A_{t} .
$$

Since both $u\left(X_{t}\right)-u\left(X_{0}\right)$ and $A_{t}$ are additive functionals, $-E^{x}\left[A_{\infty} \mid \mathscr{F}_{t}\right]-u\left(X_{0}\right)$ is also an additive functional. Thus $(1.10)$ is a decomposition of $u\left(X_{t}\right)-u\left(X_{0}\right)$ into the sum of a martingale additive functional and a continuous additive functional of zero energy. But by (1.7),

$$
u\left(X_{t}\right)-u\left(X_{0}\right)=\int_{0}^{t} \nabla u\left(X_{s}\right) \cdot d X_{s}+A_{t}^{[u]}
$$

is also such a decomposition. By Theorem 5.2.2 of [10] such a decomposition is unique, therefore we know that

$$
A_{t}=A_{t}^{[u]}
$$

and

$$
\int_{0}^{t} \nabla u\left(X_{s}\right) \cdot d X_{s}=-E^{x}\left(A_{\infty} \mid \mathscr{F}_{t}\right)-u\left(X_{0}\right)=u\left(X_{t}\right)-u\left(X_{0}\right)-A_{t} .
$$


Thus

$$
\begin{aligned}
E^{x} \int_{0}^{t}\left|\nabla u\left(X_{s}\right)\right|^{2} d s & =E^{x}\left(\int_{0}^{t} \nabla u\left(X_{s}\right) \cdot d X_{s}\right)^{2} \\
& \leqq 2 E^{x}\left(u\left(X_{t}\right)-u\left(X_{0}\right)\right)^{2}+2 E^{x} A_{t}^{2} .
\end{aligned}
$$

It is very easy to show that

$$
\lim _{t \downarrow 0 x \in R^{d}} \sup ^{x} A_{t}^{2}=0 .
$$

Therefore it follows by (1.2) that to show $u$ is admissible, we need only to show that

$$
\lim _{t \downarrow 0} \sup _{x \in R^{d}} E^{x}\left(u\left(X_{t}\right)-u\left(X_{0}\right)\right)^{2}=0 \text {. }
$$

For any $\varepsilon>0$, there exist an $r>0$ such that $|u(x)-u(y)|<\sqrt{\varepsilon / 2}$ whenever $|x-y| \leqq r$ since $u$ is uniformly continuous. For this fixed $r$,

$$
\lim _{t \downarrow 0} P^{0}\left\{\left|X_{t}\right|>r\right\}=0
$$

thus there exists a $t_{0}>0$ such that

$$
P^{0}\left\{\left|X_{t}\right|>r\right\}<\frac{\varepsilon}{8\|u\|_{\infty}^{2}},
$$

whenever $t<t_{0}$. Consequently

$$
\begin{aligned}
& E^{x}\left(u\left(X_{t}\right)-u\left(X_{0}\right)\right)^{2} \\
& \leqq 4\|u\|_{\infty}^{2} P^{x}\left\{\left|X_{t}-X_{0}\right|>r\right\}+E^{x}\left\{\left|X_{t}-X_{0}\right| \leqq r ;\left(u\left(X_{t}\right)-u\left(X_{0}\right)\right)^{2}\right\} \\
& =4\|u\|_{\infty}^{2} P^{0}\left\{\left|X_{t}\right|>r\right\}+E^{x}\left\{\left|X_{t}-X_{0}\right| \leqq r ;\left(u\left(X_{t}\right)-u\left(X_{0}\right)\right)^{2}\right\}<\varepsilon,
\end{aligned}
$$

whenever $t<t_{0}$. The proof is now complete.

\section{Additive functionals generated by admissible functions}

From now on we are going to fix an admissible function $u$. We shall write $A_{t}$ in place of $A_{t}^{[u]}$ when there is no confusion. Define

$$
e(t)=\exp \left(A_{t}^{[u]}\right) \text {. }
$$

Then $(e(t))_{t \geqq 0}$ is a continuous multiplicative functional of $X$.

(2.1) Theorem. There exists a $t_{0}>0$ such that

$$
\sup _{x \in R^{d}} E^{x}\left\{\sup _{t \leqq t_{0}} e(t)\right\}<\infty \text {. }
$$

Proof. For any $x$,

$$
\begin{aligned}
E^{x}\left\{\sup _{t \leqq t_{0}} e(t)\right\} & =E^{x}\left\{\sup _{t \leqq t_{0}} \exp \left(u\left(X_{t}\right)-u\left(X_{0}\right)-\int_{0}^{t} \nabla u\left(X_{s}\right) \cdot d X_{s}\right)\right\} \\
& \leqq e^{2\|u\|_{\infty}} E^{x}\left\{\sup _{t \leqq t_{0}} \exp \left(-\int_{0}^{t} \nabla u\left(X_{s}\right) \cdot d X_{s}\right)\right\}
\end{aligned}
$$


Therefore we need only to show that there exists a $t_{0}>0$ such that

$$
\sup _{x \in R^{d}} E^{x}\left\{\sup _{t \leqq t_{0}} \exp \left(-\int_{0}^{t} \nabla u\left(X_{s}\right) \cdot d X_{s}\right)\right\}<\infty .
$$

For any $t>0$,

$$
\begin{aligned}
E^{x}\left\{\left|\int_{0}^{t} \nabla u\left(X_{s}\right) \cdot d X_{s}\right|\right\} & \leqq\left(E^{x}\left\{\left(\int_{0}^{t} \nabla u\left(X_{s}\right) \cdot d X_{s}\right)^{2}\right\}\right)^{\frac{1}{2}} \\
& =\left(E^{x} \int_{0}^{t}\left|\nabla u\left(X_{s}\right)\right|^{2} d s\right)^{\frac{1}{2}} .
\end{aligned}
$$

Since $u$ is admissible, we know that there exists a $t_{0}>0$ such that for any $t \leqq t_{0}$,

$$
\sup _{x \in R^{d}} E^{x} \int_{0}^{t}\left|\nabla u\left(X_{s}\right)\right|^{2} d s \leqq c^{2}<\frac{1}{16},
$$

consequently, for any $t \leqq t_{0}$,

$$
\sup _{x \in R^{d}} E^{x}\left\{\left|\int_{0}^{t} \nabla u\left(X_{s}\right) \cdot d X_{s}\right|\right\} \leqq c<\frac{1}{4} .
$$

Now for any stopping times $S \leqq T \leqq t_{0}$,

$$
\begin{aligned}
& E^{x}\left\{\mid \int_{s}^{T} \nabla u\left(X_{s}\right) \cdot d X_{s} \| \mathscr{F}_{s}\right\} \\
\leqq & \left(E^{x}\left\{\left(\int_{S}^{T} \nabla u\left(X_{s}\right) \cdot d X_{s}\right)^{2} \mid \mathscr{F}_{s}\right\}\right)^{\frac{1}{2}} \\
= & \left(E^{x}\left\{\int_{s}^{T}\left|\nabla u\left(X_{s}\right)\right|^{2} d s \mid \mathscr{F}_{s}\right\}\right)^{\frac{1}{2}} \\
\leqq & \left(E^{x}\left\{\int_{s}^{t_{0}+s}\left|\nabla u\left(X_{s}\right)\right|^{2} d s \mid \mathscr{F}_{S}\right\}\right)^{\frac{1}{2}} \\
= & \left(E^{X_{s}} \int_{0}^{t_{0}}\left|\nabla u\left(X_{s}\right)\right|^{2} d s\right)^{\frac{1}{2}} \\
\leqq & c<\frac{1}{4} .
\end{aligned}
$$

Therefore by the John-Nirenberg inequality (see [8]) we know that

$$
\sup _{x \in R^{d}} E^{x}\left\{\exp \left(\sup _{t \leqq t_{0}}\left|\int_{0}^{t} \nabla u\left(X_{s}\right) \cdot d X_{s}\right|\right)\right\} \leqq \frac{1}{1-4 c},
$$

consequently

$$
\sup _{x \in R^{d}} E^{x}\left\{\sup _{t \leq t_{0}} \exp \left(-\int_{0}^{t} \nabla u\left(X_{s}\right) \cdot d X_{s}\right)\right\}<\infty .
$$

The proof is now complete.

An immediate consequence of (2.1) is the following 
(2.2) Corollary. There exist $C>0$ and $\beta>1$ such that

$$
\sup _{x \in R^{a}} E^{x}\{e(t)\} \leqq C e^{\beta t}
$$

Another consequence of (2.1) is the following:

(2.3) Corollary. For any $t>0$,

$$
\sup _{x \in R^{d}} E^{x}\left\{\sup _{s \leqq t} e(s)\right\}<\infty .
$$

Proof. For any $t>0$, there exists an integer $n$ such that $t \leqq n t_{0}$, where $t_{0}$ is specified in (2.1). Then

$$
\begin{aligned}
\sup _{x \in R^{d}} E^{x}\left\{\sup _{s \leqq t} e(s)\right\} & \leqq \sup _{x \in R^{d}} E^{x}\left\{\sup _{s \leqq n t_{0}} e(s)\right\} \\
& \leqq \sum_{k=0}^{n-1} \sup _{x \in R^{d}} E^{x}\left\{\sup _{k t_{0}<s \leqq(k+1) t_{0}} e(s)\right\} \\
& =\sum_{k=0}^{n-1} \sup _{x \in R^{d}} E^{x}\left\{e\left(k t_{0}\right) E^{X\left(k t_{0}\right)}\left(\sup _{s \leqq t_{0}} e(s)\right)\right\} \\
& \leqq C_{1} \sum_{k=0}^{n-1} \sup _{x} E^{x}\left\{e\left(k t_{0}\right)\right\} \\
& \leqq C_{1} \sum_{k=0}^{n-1} C e^{k \beta t_{0}}<\infty
\end{aligned}
$$

where $C_{1}=\sup _{x \in R^{a}} E^{x}\left\{\sup _{s \leq t_{0}} e(s)\right\}$ and $C$ and $\beta$ are specified by (2.2).

(2.4) Theorem. If $D$ is a bounded domain in $R^{d}$, then for any $\varepsilon>0$ there exists a $t_{0}>0$ such that

$$
\sup _{x \in R^{d}} E^{x}\left\{\sup _{t \leqq t_{0}} e^{A(t \wedge \tau D)}\right\}<1+\varepsilon .
$$

Proof. Since only the restriction of $u$ on the closure of $D$ is involved in $A\left(t \wedge \tau_{D}\right)$, we can assume that $u$ has compact support in $R^{d}$.

For any $x$,

$$
\begin{aligned}
& E^{x}\left\{\sup _{t \leqq t_{0}} e^{A(t \wedge \tau)}\right\} \\
& \leqq E^{x}\left\{\sup _{t \leqq t_{0}} e(t)\right\} \\
& =E^{x}\left\{\sup _{t \leqq t_{0}} \exp \left(u\left(X_{t}\right)-u\left(X_{0}\right)-\int_{0}^{t} \nabla u\left(X_{s}\right) \cdot d X_{s}\right)\right\} \\
& \leqq\left(E^{x}\left\{\sup _{t \leqq t_{0}} \exp \left(2\left|u\left(X_{t}\right)-u\left(X_{0}\right)\right|\right)\right\}\right)^{\frac{1}{2}}\left(E^{x}\left\{\sup _{t \leqq t_{0}} \exp \left(-2 \int_{0}^{t} \nabla u\left(X_{s}\right) \cdot d X_{s}\right)\right\}\right)^{\frac{1}{2}} .
\end{aligned}
$$


Therefore we need only to show that

$$
\lim _{t_{0} \downarrow 0 x \in R^{d}} \sup _{x \in t_{0}} E^{x}\left\{\sup _{t \leqq} \exp \left(2\left|u\left(X_{t}\right)-u\left(X_{0}\right)\right|\right)\right\}=1,
$$

and

$$
\lim _{t_{0} \downarrow 0} \sup _{x \in R^{d}} E^{x}\left\{\sup _{t \leqq t_{0}} \exp \left(-2 \int_{0}^{t} \nabla u\left(X_{s}\right) \cdot d X_{s}\right)\right\}=1 .
$$

We are going to prove (2.6) first. Since $u$ is admissible, we know that for any $0<\delta<\frac{1}{4}$ there exists a $t_{0}>0$ such that for any $\mathrm{t} \leqq t_{0}$,

$$
\sup _{x \in \boldsymbol{R}^{a}} E^{x} \int_{0}^{t} 4\left|\nabla u\left(X_{s}\right)\right|^{2} d s \leqq \delta^{2} .
$$

Repeating part of the proof of Theorem 2.1 and applying the John-Nirenberg inequality we get

$$
\sup _{x \in R^{d}} E^{x}\left\{\sup _{t \leqq t_{0}} \exp \left(-2 \int_{0}^{t} \nabla u\left(X_{s}\right) \cdot d X_{s}\right)\right\} \leqq \frac{1}{1-4 \delta},
$$

from which (2.6) follows.

Now, we are going to prove (2.5). For any $\eta>0$ there exists an $r>0$ such that $|u(x)-u(y)| \leqq \eta$ whenever $|x-y| \leqq r$ since $u$ is uniformly continuous. For this fixed $r$, we have

$$
\lim _{t \downarrow 0} \sup _{x \in R^{d}} P^{x}\left\{\tau_{B(x, r)} \leqq t\right\}=\lim _{t \downarrow 0} P^{0}\left\{\tau_{B(0, r)} \leqq t\right\}=0,
$$

where $B(x, r)$ denote the ball of radius $r$ around $\mathrm{x}$. Thus there exists a $t_{0}>0$ such that

$$
\sup _{x \in R^{d}} P^{x}\left\{\tau_{B(x, r)} \leqq t_{0}\right\} \leqq \eta
$$

Consequently for this $t_{0}$ we have

$$
\begin{aligned}
& \sup _{x \in R^{d}} E^{x}\left\{\sup _{t \leqq t_{0}} \exp \left(2\left|u\left(X_{t}\right)-u\left(X_{0}\right)\right|\right)\right\} \\
& \leqq e^{4\|u\|_{\infty}} \sup _{x \in R^{d}} P^{x}\left\{\tau_{B(x, r)} \leqq t_{0}\right\}+e^{2 \eta} \sup _{x \in R^{d}} E^{x}\left\{\tau_{B(x, r)}>t_{0}\right\} \\
& \leqq \eta e^{4\|u\|_{\infty}}+e^{2 \eta}
\end{aligned}
$$

From this (2.5) follows.

(2.7) Theorem. Let $D$ be a bounded domain in $R^{d}$ and fix $\varepsilon>0$. There exists $a \delta>0$ such that for any Borel subset $B$ of $D$ whose Lebesgue measure is less than $\delta$,

$$
\sup _{x \in R^{d}} E^{x}\left\{e\left(\tau_{B}\right)\right\}<1+\varepsilon .
$$

Proof. For any $\eta>0$, from Theorem 2.4 we know that we can choose a $t_{0}>0$ such that

$$
\sup _{x \in R^{d}} E^{x}\left\{\sup _{t \leqq t_{0}} \exp \left(A\left(t \wedge \tau_{D}\right)\right)\right\} \leqq 1+\eta
$$


and

$$
\sup _{x \in R^{d}} E^{x}\left\{\sup _{t \leqq t_{0}} \exp \left(2 A\left(t \wedge \tau_{D}\right)\right)\right\} \leqq(1+\eta)^{2} .
$$

In particular, (2.8) implies that

$$
\sup _{x \in R^{d}} E^{x}\left\{t_{0}<\tau_{D}, \exp \left(2 A\left(t_{0}\right)\right)\right\} \leqq(1+\eta)^{2} .
$$

By using the Markov property, we can get that for any positive integer $n$,

$$
\sup _{x \in R^{d}} E^{x}\left\{n t_{0}<\tau_{D}, \exp \left(2 A\left(n t_{0}\right)\right)\right\} \leqq(1+\eta)^{2 n}
$$

For any $x \in R^{d}$, if $P^{x}\left(\tau_{B}=0\right)=1$, then $E^{x}\left\{e\left(\tau_{B}\right)\right\}=1$. Otherwise $P^{x}\left(\tau_{B}=0\right)=0$ and we have

$$
\begin{aligned}
E^{x}\left\{e\left(\tau_{B}\right)\right\} & =\sum_{n=0}^{\infty} E^{x}\left\{n t_{0}<\tau_{B} ; e\left(n t_{0}\right) E^{X\left(n t_{0}\right)}\left\{0<\tau_{B} \leqq 1 ; e\left(\tau_{B}\right)\right\}\right\} \\
& \leqq \sum_{n=0}^{\infty} E^{x}\left\{n t_{0}<\tau_{B} ; e\left(n t_{0}\right) \sup _{x \in R^{d}} E^{x}\left\{\sup _{t \leqq 1} e^{A\left(t_{\wedge} \tau_{D}\right)}\right\}\right\} \\
& \leqq(1+\eta) \sum_{n=0}^{\infty} E^{x}\left\{n t_{0}<\tau_{B} ; e\left(n t_{0}\right)\right\} \\
& \leqq(1+\eta) \sum_{n=0}^{\infty}\left(P^{x}\left(n t_{0}<\tau_{B}\right)\right)^{\frac{1}{2}}\left(E^{x}\left\{n t_{0}<\tau_{D} ; \exp \left(2 A\left(n t_{0}\right)\right)\right\}\right)^{\frac{1}{2}} \\
& \leqq(1+\eta) \sum_{n=0}^{\infty}(1+\eta)^{n}\left(P^{x}\left(n t_{0}<\tau_{B}\right)\right)^{\frac{1}{2}}
\end{aligned}
$$

Choose a $\beta>0$ such that $e^{-\beta}(1+\eta)<1$. We know that there exists a $\delta>0$ such that if the Lebesgue measure of $B$ is less than $\delta$, then

$$
\sup _{x \in R^{d}} P^{x}\left(1<\tau_{B}\right) \leqq e^{-2 \beta}
$$

It follows by the Markov property that

$$
P^{x}\left(n<\tau_{B}\right) \leqq e^{-2 n \beta} .
$$

Using this we get

$$
\sup _{x \in R^{d}} E^{x}\left\{e\left(\tau_{B}\right)\right\} \leqq \frac{1+\eta}{1-\left(e^{-\beta}(1+\eta)\right)},
$$

from which the conclusion of the theorem follows.

\section{The gauge theorem}

In this section we fix a bounded, connected open subset $D$ of $R^{d}$. If we define

$$
g(x)=E^{x}\left\{e\left(\tau_{D}\right)\right\} \quad \forall x \in R^{d} .
$$

then $g$ is called the gauge function for $(D, u)$. 
For any integer $n>0$ and any $x \in R^{d}$, put

$$
g_{n}(x)=E^{x}\left\{e\left(\tau_{D}\right) \wedge n\right\} .
$$

Clearly, for each $n>0, g_{n}$ is a bounded universally measurable function and

$$
g_{n}(x) \uparrow g(x) .
$$

The following result is not directly related to the gauge theorem, but we will use it to derive some consequences of the boundedness of the gauge function.

(3.2) Theorem. $\inf _{x} g(x)>0$.

Proof. Since $u$ is admissible, we know that the function

$$
E^{x} \int_{0}^{\tau D}\left|\nabla u\left(X_{t}\right)\right|^{2} d t
$$

is bounded by a constant $M$ on $R^{d}$. Then we have

$$
\begin{aligned}
E^{x}\left|\int_{0}^{\tau D} \nabla u\left(X_{t}\right) \cdot d X_{t}\right| & \leqq\left(E^{x}\left(\int_{0}^{t D} \nabla u\left(X_{t}\right) \cdot d X_{t}\right)^{2}\right)^{\frac{1}{2}} \\
& =\left(E^{x} \int_{0}^{\tau D}\left|\nabla u\left(X_{t}\right)\right|^{2} d t\right)^{\frac{1}{2}} \\
& \leqq \sqrt{M}
\end{aligned}
$$

By Jensen's inequality

$$
\begin{aligned}
g(x) & =E^{x}\left\{\exp \left(u\left(X_{\tau D}\right)-u\left(X_{0}\right)-\int_{0}^{\tau_{D}} \nabla u\left(X_{s}\right) \cdot d X_{s}\right)\right\} \\
& \geqq e^{-2\|u\|_{\infty}} E^{x}\left\{\exp \left(-\left|\int_{0}^{\tau_{D}} \nabla u\left(X_{s}\right) \cdot d X_{s}\right|\right)\right\} \\
& \geqq e^{-2\|u\|_{\infty}} \exp \left(-E^{x}\left|\int_{0}^{\tau_{D}} \nabla u\left(X_{s}\right) \cdot d X_{s}\right|\right) \\
& \geqq e^{-2\|u\|_{\infty}-\sqrt{M}} .
\end{aligned}
$$

Thus the assertion is true.

(3.3) Theorem. $g$ is lower semi-continuous with respect to the fine topology.

Proof. It follows immediately from (3.1) and the two results below.

(3.4) Lemma. For each $n>0, g_{n}$ is Borel measurable.

Proof. For any $t>0$ and any $x \in R^{d}$, define

$$
h_{n, t}(x)=E^{x}\left\{\exp \left(A_{\tau_{D}} \circ \theta_{t}\right) \wedge n\right\} .
$$

Since

$$
h_{n, t}(x)=E^{x} g_{n}\left(X_{t}\right),
$$


we know that $h_{n, t}(\cdot)$ is continuous on $R^{d}$. Since

$$
\lim _{t \downarrow 0}\left(t+\tau_{D} \circ \theta_{t}\right)=\tau_{D},
$$

by the dominated convergence theorem we know that

$$
\begin{aligned}
\lim _{t \downarrow 0} h_{n, t}(x) & =\lim _{t \downarrow 0} E^{x}\left\{\exp \left(A_{t+\tau_{D} \circ \theta_{t}}-A_{t}\right) \wedge n\right\} \\
& =g_{n}(x),
\end{aligned}
$$

where in the first equality above we used the fact $A_{\tau_{D}} \circ \theta_{t}=A_{t+\tau \text { o } \theta_{t}}-A_{t}$. Hence $g_{n}$ is Borel measurable.

(3.5) Lemma. For each $n>0, g_{n}$ is finely continuous.

Proof. Fix an $n>0$. It follows from (3.4) that $\left\{g_{n}\left(X_{\varepsilon}\right) ; t \geqq 0\right\}$ is a bounded optional process. For every decreasing sequence $\left(T_{k}\right)$ of bounded stopping times with limit $T$,

$$
\lim _{k \rightarrow \infty}\left(T_{k}+\tau_{D} \circ \theta_{T_{k}}\right)=T+\tau_{D} \circ \theta_{T},
$$

thus by the dominated convergence theorem

$$
\begin{aligned}
\lim _{k \rightarrow \infty} E^{x}\left\{g_{n}\left(X_{T_{k}}\right)\right\} & =\lim _{k \rightarrow \infty} E^{x}\left\{\exp \left(A_{\tau_{D}} \circ \theta_{T_{k}}\right) \wedge n\right\} \\
& =\lim _{k \rightarrow \infty} E^{x}\left\{\exp \left(A_{T_{k}+\tau_{D} \circ \theta_{T_{k}}}-A_{T_{k}}\right) \wedge n\right\} \\
& =E^{x}\left\{\exp \left(A_{T+\tau_{D} \circ \theta_{T}}-A_{T}\right) \wedge n\right\} \\
& =E^{x}\left\{\exp \left(A_{\tau_{D}} \circ \theta_{T}\right) \wedge n\right\} \\
& =E^{x}\left\{g\left(X_{T}\right)\right\}
\end{aligned}
$$

where in the second and fourth equalities above we used the facts $A_{\tau_{\nu}} \circ \theta_{T_{k}}=A_{T_{k}+\tau_{0} \theta_{T_{k}}}-A_{T_{k}}$ and $A_{\tau_{b}} \circ \theta_{T}=A_{T+\tau_{D} \theta_{T}}-A_{T}$ respectively. Therefore by Theorem VI.48 of [8] we know that $\left\{g_{n}\left(X_{t}\right) ; t \geqq 0\right\}$ is a right continuous process, consequently $g_{n}$ is finely continuous.

With our preparations so far we can prove the following result which is the counterpart of Theorem 1 of [7]. In fact, the proof there carries over to our case with one minor modification. We give the complete proof here for the reader's convenience.

(3.6) Theorem. The set

$$
B=\{x \in D ; g(x)<\infty\}
$$

is absorbing with respect to the Brownian motion killed outside $D$, namely, for any $x \in B, P^{x}\left(\tau_{B}<\tau_{D}\right)=0$. Moreover, $g$ is actually bounded on $B$.

Proof. Let $x \in B$ and let $K$ be any compact subset of $D \backslash B$. By the strong Markov property

$$
E^{x}\left\{e\left(T_{K}\right) g\left(X\left(T_{K}\right)\right) ; T_{K}<\tau_{D}\right\} \leqq g(x)<\infty,
$$

where $T_{K}=\inf \{t>0: X(t) \in K\}$. Since $K$ is closed, $X\left(T_{K}\right) \in K$, thus $g\left(X\left(T_{K}\right)\right)$ $=\infty$ on $\left\{T_{K}<\tau_{D}\right\}$. On the other hand, $e\left(T_{K}\right)$ is strictly positive. It follows from 
(3.7) that $P^{x}\left(T_{K}<\tau_{D}\right)=0$. This being true for all compact subsets $K$ of $D \backslash B$, we conclude that

$$
P^{x}\left\{T_{D \backslash B}<\tau_{D}\right\}=0 .
$$

Thus $B$ is absorbing as asserted.

Choose $N$ so large that the Lebesgue measure of

$$
L=D \cap\{N<g<\infty\}
$$

is less than the $\delta$ specified in (2.7). Then we have that if $x \in L$,

$$
g(x)=E^{x}\left\{e\left(\tau_{L}\right) ; \tau_{L}=\tau_{D}\right\}+E^{x}\left\{e\left(\tau_{L}\right) g\left(X\left(\tau_{L}\right)\right) ; \tau_{L}<\tau_{D}\right\} .
$$

It follows from Theorem 2.7 that the first term on the right hand side is bounded by a constant $C$. Because $B$ is absorbing, $X\left(\tau_{L}\right)$ does not belong to $D \backslash B P^{x}$-a.s. on $\left\{\tau_{L}<\tau_{D}\right\}$, hence it must belong to the fine closure of $\{g \leqq N\}$. Since $g$ is finely lower semi-continuous by Theorem 3.3, this fine closure is just $\{g \leqq N\}$. Therefore the second term on the right hand side of the identity above is bounded by $C N$. It follows that on $L, g$ is bounded by $C+C N$. On $B \backslash L, g$ is bounded by $N$. The proof is now complete.

Remark. If in the definition of $B, D$ is replaced by $D^{*}$, where

$$
D^{*}=\left\{x \in R^{d}: P^{x}\left(\tau_{D}>0\right)=1\right\},
$$

the conclusion of Theorem 3.6 is still true. Of course, some minor changes are needed in the proof.

(3.8) The gauge theorem. $g$ is either identically infinite on $D$ or $g$ is bounded on $R^{d}$.

Proof. We have proved in Theorem 3.6 that there exists a constant $C$ such that $B=\{g \leqq C\}$. Hence $B$ is finely closed and $D \backslash B$ is finely open. If $D \backslash B$ is not empty, choose $x_{0} \in D \backslash B$; then $E^{x_{0}} \int_{0}^{\tau_{D}} 1_{D \backslash B}\left(X_{t}\right) d t>0$. Since $D$ is connected, $E^{x} \int_{0}^{\tau_{D}} 1_{D \backslash B}\left(X_{t}\right) d t>0$ for every $x \in D$, which is impossible by Theorem 3.6 unless $B$ is empty. Therefore either $B$ or $D \backslash B$ is empty. If $D \backslash B$ is empty, then $g$ is bounded on $D=B$. To see $g$ is bounded on $R^{d}$, let $x \in \partial D$ and not regular for $D^{c}$. Then

$$
\begin{aligned}
g(x) & =\lim _{n \rightarrow \infty} g_{n}(x) \\
& =\lim _{n \rightarrow \infty} \lim _{t \downarrow 0} E^{x}\left\{(e(t) \wedge n) g_{n}\left(X_{t}\right) ; t<\tau_{D}\right\} \\
& \leqq C \lim _{t \downarrow 0} E^{x}\left\{e(t) ; t<\tau_{D}\right\} \\
& \leqq C \sup _{x \in R^{d}} E^{x}\left\{\sup _{t \leqq 1} e(t)\right\}
\end{aligned}
$$

since $g \leqq C$ in $D$. For $x$ regular for $D^{c}, g(x)=1$. Hence $g$ is bounded in $R^{d}$. Finally, of course, if $B$ is empty, $g$ is infinite on $D$.

Remark. Again $D$ can be replaced by $D^{*}$ in the theorem above.

Now we are going to give some important consequences of the boundedness of the gauge function. But first we give a lemma which will be needed below. 
(3.9) Lemma. If $g$ is bounded, then for any $\delta>0$, there exists a constant $C(\delta)$ such that

$$
\|g\|_{\infty}^{-1} g(x) \leqq \sum_{n=0}^{\infty} E^{x}\left\{n \delta<\tau_{D} ; e(n \delta)\right\} \leqq C(\delta) .
$$

Proof. Similar to that Lemma 9 of [7].

The following assertion looks much stronger than the assumption that the gauge function is bounded.

(3.10) Theorem. If the gauge function $g$ is bounded, then

$$
\sup _{x \in R^{d}} E^{x}\left\{\sup _{t \leqq \tau_{D}} e(t)\right\}<\infty .
$$

Proof. For any $x$, we have

$$
\begin{aligned}
E^{x}\left\{\sup _{t \leqq \tau_{D}} e(t)\right\} & \leqq \sum_{n=0}^{\infty} E^{x}\left\{\sup _{n \wedge \tau_{D}<t \leqq(n+1) \wedge \tau_{D}} e(t)\right\} \\
& \leqq \sum_{n=0}^{\infty} E^{x}\left\{n<\tau_{D} ; e(n) E^{X(n)}\left\{\sup _{t \leqq 1} e(t)\right\}\right\} \\
& \leqq C \sum_{0}^{\infty} E^{x}\left\{n<\tau_{D} ; e(n)\right\} \\
& \leqq C \cdot C(1),
\end{aligned}
$$

where $C=\sup _{x \in R^{d}} E^{x}\left\{\sup _{t \leqq 1} e(t)\right\}$ and $C(1)$ is specified by (3.9). Thus the assertion is true.

(3.11) Theorem. Suppose that a Radon measure $v$ is in $K_{d}$ and that $B_{t}$ is the positive continuous additive functional associated with $v$. If $g$ is bounded, then

$$
\sup _{x \in R^{a}} E^{x} \int_{0}^{\tau} D(t) d B_{t}<\infty .
$$

Proof. Since $u$ is admissible and $v \in K_{d}$, we know that

$$
\begin{aligned}
\sup _{x \in R^{d}} E^{x} \int_{0}^{t D \wedge 1} e(t) d B_{t} & \leqq \sup _{x \in R^{d}} E^{x}\left\{B(1) \sup _{t \leqq 1} e(t)\right\} \\
& \leqq\left(\sup _{x \in R^{d}} E^{x} B^{2}(1)\right)^{\frac{1}{2}}\left(\sup _{x \in R^{d}} E^{x}\left\{\sup _{t \leqq 1} e^{2 A_{t}}\right\}\right)^{\frac{1}{2}} \\
& =\mathrm{C}<\infty .
\end{aligned}
$$

Therefore for any $x$,

$$
\begin{aligned}
E^{x} \int_{0}^{\tau_{D}} e(t) d B_{t} & =\sum_{n=0}^{\infty} E^{x}\left\{n<\tau_{D} ; \int_{n}^{\tau_{D} \wedge(n+1)} e(t) d B_{t}\right\} \\
& =\sum_{n=0}^{\infty} E^{x}\left\{n<\tau_{D} ; e(n) E^{X(n)} \int_{0}^{\tau_{D} \wedge 1} e(t) d B_{t}\right\}
\end{aligned}
$$




$$
\begin{aligned}
& \leqq C \sum_{n=0}^{\infty} E^{x}\left\{n<\tau_{D} ; e(n)\right\} \\
& \leqq C \cdot C(1),
\end{aligned}
$$

where $C(1)$ is specified by (3.9). Thus the assertion is true.

The following result gives several properties that are equivalent to the boundedness of the gauge function. It is counterpart of Theorem 6 of [7], and the proof there can carry through to our case with some minor modifications.

(3.12) Theorem. Any one of the following properties is equivalent to the boundedness of the gauge function $g$.

(a) For some $\delta>0$ and some $x \in D$,

$$
\sum_{n=0}^{\infty} E^{x}\left\{n \delta<\tau_{D} ; e(n \delta)\right\}<\infty .
$$

(b) For all $\delta>0$ and all $x \in D,(3.13)$ is true.

(c) For some $x \in D$,

$$
E^{x} \int_{0}^{\tau_{D}} e(t) d t<\infty
$$

(d) There exists a $t>0$ such that

$$
\sup _{x \in D} E^{x}\left\{t<\tau_{D} ; e(t)\right\}<1 \text {. }
$$

(e) There exist $C>0$ and $b>0$ such that

$$
\sup _{x \in D} E^{x}\left\{t<\tau_{D} ; e(t)\right\} \leqq C e^{-b t} .
$$

Proof. Omitted.

Acknowledgement. We would like to thank an anonymous referee whose comments lead to some improvements of the earlier version of this paper.

\section{References}

1. Aizenman, N., Simon, B.: Brownian motion and Harnack inequality for Schrödinger operators. Commun. Pure Appl. Math. 35, 209-273 (1982)

2. Blanchard, $\mathrm{Ph}$., $\mathrm{Ma}, \mathrm{Z}$.: Semigroup of Schrödinger operators with potentials given by radon measures. In: Albevrio, S. (ed.) Proc. 2nd Ascona, Locarno, Como Meeting. Singapore: World Scientific (to appear)

3. Blanchard, Ph., Ma. Z.: New results on the Schrödinger semigroups with potentials given by signed smooth measures. (Lect. Notes Math., Vol. 1444) Berlin Heidelberg New York: Springer 1990

4. Blumenthal, R.M., Getoor, R. K.: Markov processes and potential theory. New York: Academic Press 1968

5. Cranston, M., Fabes, E., Zhao, Z.: Conditional gauge and potential theory for Schrödinger operators. Trans. Am. Math. Soc. 307, 171-194 (1988)

6. Chung, K.L., Rao, K.M.: Feynman-Kac functional and the Schrödinger equation. In: Cinlar, E., Chung, K.L., Getoor, R.K. (eds.) Seminar on stochastic processes, pp. 1-30. Boston: Birkhäuser 1981 
7. Chung, K.L., Rao, K.M.: General gauge theorem for multiplicative functionals. Trans. Am. Math. Soc. 306, 819-836 (1988)

8. Dellacherie, C., Meyer, P.A.: Probabilities et potentiel: théorie des martingales. Paris: Herman 1980

9. Falkner, N.: Feynman-Kac functional and positive solutions of $\frac{1}{2} A u+q u=0$. Z. Wahrscheinlichkeitstheor. Verw. Geb. 65, 19-35 (1983)

10. Fukushima, M.: Dirichlet forms and Markov processes. Amsterdam: North-Holland 1980

11. Ma, Z., Song, R.: Probabilistic methods in Schrödinger equations. In: Cinlar, E., Chung, K. L., Getoor, R. K. (eds.) Seminar on stochastic processes, pp. 135-164. Boston: Birkhäuser 1990

12. Revuz, D.: Mesures associees aux fonctionnelles additives de Markov. 1. Trans. Am. Math. Soc. 148, 501-531 (1970)

13. Sturm, K.T.: Gauge theorems for resolvents with applications to Markov processes. Probab. Theory Relat. Fields 89, 387-406 (1991)

14. Zhao, Z.: Uniform boundedness of conditional gauge and Schrödinger equations. Commun. Math. Phys. 93, 19-31 (1983) 